\title{
Bronchial thermoplasty in asthma: current perspectives
}

\author{
This article was published in the following Dove Press journal: \\ Journal of Asthma and Allergy \\ 15 May 2015 \\ Number of times this article has been viewed
}

\section{Balaji Laxmanan \\ D Kyle Hogarth}

Section of Pulmonary and Critical Care Medicine, University of Chicago Medicine, Chicago, IL, USA
Correspondence: Balaji Laxmanan; D Kyle Hogarth

Section of Pulmonary and Critical Care Medicine, University of Chicago Medicine, 584I S Maryland Ave, MC 6076, Chicago, IL 60637, USA Tel + I 773702 I806; + I 7737024773 Email balaji.laxmanan@uchospitals.edu; dhogarth@uchicago.edu
Abstract: Bronchial thermoplasty (BT) is a novel therapy for patients with severe asthma. Using radio frequency thermal energy, it aims to reduce the airway smooth muscle mass. Several clinical trials have demonstrated improvements in asthma-related quality of life and a reduction in the number of exacerbations following treatment with BT. In addition, recent data has demonstrated the long-term safety of the procedure as well as sustained improvements in rates of asthma exacerbations, reduction in health care utilization, and improved quality of life. Further study is needed to elucidate the underlying mechanisms that result in these improvements. In addition, improved characterization of the asthma subphenotypes likely to exhibit the largest clinical benefit is a critical step in determining the precise role of BT in the management of severe asthma.

Keywords: bronchial thermoplasty, severe asthma, airway smooth muscle

\section{Introduction}

Asthma affects more than 235 million people worldwide and more than 25 million people in the United States alone. ${ }^{1,2}$ It is a disease caused by chronic inflammation of the large and small airways resulting in airway hyper-responsiveness and excessive mucous secretion. Clinically, this manifests as wheezing, shortness of breath, cough, and airflow obstruction. Asthma treatment centers on reducing inflammation with inhaled corticosteroids (ICS) and relaxing airway smooth muscle (ASM) with inhaled bronchodilators along with minimizing exposure to allergic triggers and modifying the allergic response. While the majority of patients will achieve symptom control with the regular use of these medications, a subpopulation will have persistent symptoms despite maximal medical therapy. These severe asthmatics only encompass $5 \%-10 \%$ of all asthma patients, but require a disproportionate quantity of health care resources. ${ }^{3-5}$

The definition of severe asthma is complex and involves an assessment of asthma symptoms, rescue short-acting bronchodilator use, pulmonary function, the requirement for and dosing of controller medications, and the number, severity, and risk of exacerbations. The American Thoracic Society and European Respiratory Society define severe asthma as requiring treatment with high-dose ICS and a second controller medication to maintain asthma control. ${ }^{6}$ Additionally, patients who had required systemic corticosteroids for more than $50 \%$ of the previous year are also classified as severe asthmatics. While these definitions help categorize patients and identify this subpopulation, it is important to recognize that severe asthma is a heterogeneous condition with multiple subphenotypes. ${ }^{7}$ 
Unfortunately, therapeutic options for patients with severe asthma are limited. Adjunctive therapies targeting other mediators of the inflammatory pathway have yielded variable results. Antileukotriene agents are recommended as add-on therapy for patients who remain symptomatic despite the use of high-dose ICS and long-acting beta agonists (LABAs). ${ }^{8}$ While the leukotriene receptor antagonists (LTRA) montelukast and zafirlukast appear to be effective in patients with aspirin or nonsteroidal anti-inflammatory drug (NSAID) sensitivity, ${ }^{9}$ their benefit in all patients with severe asthma is less convincing. Whereas one study showed improved asthma control and forced expiratory volume in 1 second $\left(\mathrm{FEV}_{1}\right)$ in patients already using high-dose ICS, another found no benefit when added to a regimen of ICS and another controller medication. ${ }^{10,11}$ Treatment with the 5-lipoxygenase inhibitor zileuton (Zyflo) in patients with mild to moderate asthma resulted in improved asthma control, although these patients were not treated with ICS. ${ }^{12}$ In addition, concerns over cost and the need for laboratory monitoring have limited its widespread use. Omalizumab, an anti-immunoglobulin (anti-Ig) E monoclonal antibody administered subcutaneously, has been approved for patients with moderate to severe allergic asthma. A large randomized trial in patients with severe allergic asthma showed a reduction in exacerbations, ${ }^{13}$ a finding also seen in a systematic review of 25 randomized controlled trials in patients with moderate to severe disease. ${ }^{14}$ However, the response rate to omalizumab is variable and many patients with severe asthma remain symptomatic despite this therapy.

New therapies in development for severe asthma have also focused on modulating the underlying inflammatory response. Of particular interest has been biologic therapy targeting interleukins (IL)-2, 4, 5, and $13 .{ }^{15}$ Blockade of IL-5, a potent modulator of eosinophil function and recruitment, with mepolizumab has recently been shown to improve asthma control, reduce exacerbations, and reduce oral glucocorticoid requirements in severe eosinophilic asthma. ${ }^{16,17}$ IL-4 and IL-13 are both mediators of the Th2 immune response and contribute to airway eosinophilia, mucous gland hyperplasia, and IgE production by B lymphocytes. In patients with moderate to severe asthma and peripheral blood eosinophilia, dupilumab - an anti-IL-4 receptor alpha subunit antibody was associated with reduced exacerbations upon withdrawal of ICS and LABA. ${ }^{18}$ Lebrikizumab, an anti-IL-13 antibody, was associated with short-term increases in $\mathrm{FEV}_{1}$ in patients with moderate to severe asthma, particularly in patients with elevated blood periostin levels (a surrogate marker of IL-13 activity). ${ }^{19}$ Although these therapies have yielded promising results thus far, they remain experimental and only appear effective in the subpopulation of allergic asthmatics.

\section{ASM as a target in asthma management}

Inflammation and mucous hypersecretion are key components in the pathogenesis of asthma, but the exact role of the ASM is uncertain. ASM contraction certainly causes airway narrowing and airflow obstruction in asthma. ${ }^{20}$ Further, a hallmark of asthma is an increase in ASM mass, and both hypertrophy and hyperplasia of the ASM cells is seen. ${ }^{21}$ In addition to the ASM, multiple factors contribute to the increased airways resistance and airway hyperreactivity seen in asthma, including the lung parenchyma, airway extracellular matrix, and airway-lining fluid and mucous. ${ }^{22}$ Indeed, the interplay between these various components is likely critical in determining the maximum ASM-generated tension, which simulations predict to be the single most important factor causing airway hyperreactivity. ${ }^{23}$ In addition, the ASM may directly play a pro-inflammatory role by secreting cytokines/ chemokines and modulating the migration of inflammatory cells. ${ }^{24}$ Therefore, targeting the ASM is an attractive alternative management strategy in severe asthma.

Preclinical studies in the canine airway evaluated the effects of radio frequency (RF) delivered thermal energy to the ASM. Using temperature settings of $55^{\circ} \mathrm{C}, 65^{\circ} \mathrm{C}$, and $75^{\circ} \mathrm{C}$, the airways of healthy dogs were treated using an RF catheter. There was significant improvement in airway responsiveness to methacholine in the airways treated with thermal energy at $65^{\circ} \mathrm{C}$ and $75^{\circ} \mathrm{C}$ occurring as early as 1 week following the procedure and persisting for 3 years. ${ }^{25}$ Furthermore, there was an increase in the percentage of the airway wall with altered smooth muscle with successively higher temperatures, and an inverse relationship between airway responsiveness and the percentage of altered smooth muscle. In addition, high-resolution imaging of the airways showed a significant increase in airway diameter when treated with thermal energy, both at baseline and with sequentially increasing doses of methacholine. ${ }^{26}$ Therefore, these animal models suggested that reducing the ASM mass would be a reasonable approach to reduce the bronchial hyperresponsiveness and airflow obstruction seen in patients with severe asthma.

\section{Feasibility}

Bronchial thermoplasty (BT) is the procedure of applying RF thermal energy to the airways in asthma patients with the goal of ablating the ASM. The first study of BT in human 
airways involved subjects undergoing lobectomy for known or suspected lung cancer. ${ }^{27}$ Eight patients underwent BT in the lobe to be resected approximately $2-3$ weeks prior to the operation. Using the information gained from canine studies, two of these patients were treated with thermal energy at $55^{\circ} \mathrm{C}$ and the other six were treated at $65^{\circ} \mathrm{C}$. There were no adverse events related to the thermoplasty procedure and no complications occurred during the short interim follow-up period.

On repeat bronchoscopic examination at the time of surgery, the majority of airways appeared normal. A minority exhibited expected changes including mild erythema, edema, blanching, and airway narrowing with mucous retention. Similar to that seen in the canine airway, histologic examination revealed minimal to no changes in airways treated at $55^{\circ} \mathrm{C}$. Airways treated at $65^{\circ} \mathrm{C}$ showed a pattern of epithelial sloughing and regeneration. In those patients with a longer duration between treatment and lobectomy, a return to the normal architecture was seen. Most importantly, there was a significant reduction in ASM mass of approximately $50 \%$, though there was considerable variation among patients. This important study showed that the delivery of thermal energy was effective in reducing smooth muscle mass and led to the development of large clinical trials to evaluate the efficacy and safety of this treatment in patients with asthma. It also suggested that a variable response of the ASM to thermal energy could be seen with wider clinical use.

\section{BT}

BT is a novel, minimally invasive therapeutic intervention for patients with severe persistent asthma that is uncontrolled despite the use of ICS and LABA. First approved by the United States Food and Drug Administration (FDA) in 2010, BT delivers targeted thermal energy to the airway walls with the goal of reducing ASM mass. The thermal energy is delivered using the Alair System (Boston Scientific, Natick, MA, USA). The catheter is introduced via the working channel of the flexible bronchoscope. The distal tip contains an expandable four-electrode basket, which is serially deployed in the airways. ${ }^{28}$

BT is performed in a series of three bronchoscopies. The first two sessions target the right lower lobe and left lower lobe separately while the final procedure targets the bilateral upper lobes. By convention, the right middle lobe is not treated due to concerns over causing stenosis to this typically narrow bronchus and potentially increasing the risk for chronic injury, bronchiectasis, and right middle lobe syndrome. ${ }^{29}$ Each bronchoscopy takes approximately 30-60 minutes, although this is variable and generally dependent on the experience of the endoscopist and the patient's individual bronchial anatomy as this dictates the number of activations that are delivered. Dividing the treatment into three procedures allows shorter procedure times and obviates the risks associated with widespread irritation of the airways in patients with severe asthma.

Patients are generally given systemic oral corticosteroids for 3 days preprocedure, the day of the procedure, and 1 day following the procedure to mitigate airway inflammation that may occur. In addition, an inhaled bronchodilator is given immediately prior to bronchoscopy. Our practice is to prescribe prednisone $50 \mathrm{mg}$ daily for 3 days prior to the procedure as well as on the day of bronchoscopy and the following day. Immediately preprocedure, we provide nebulized albuterol for bronchodilation and nebulized lidocaine to provide topical anesthesia and improve patient comfort.

Although the first description of the procedure in patients with asthma was performed using general anesthesia, it may be performed using local anesthesia and moderate sedation. The procedure begins with a standard examination of all airways, with particular attention to the lobe undergoing treatment. Any mucous should be cleared as this will impair visualization once thermoplasty begins. During the second and third procedures, subtle changes to the previously treated airways may be visualized and adequate healing should be ensured. The diagnostic adult bronchoscope with a minimum working channel diameter of $2.0 \mathrm{~mm}$ is used to perform the procedure. The smaller diameter of the diagnostic bronchoscope is preferred over the larger therapeutic bronchoscope to allow a more thorough visualization and treatment of the distal tracheobronchial tree.

The Alair System consists of an RF controller and a single-use catheter. The catheter is connected to the RF controller and a grounding pad is placed on the patient to complete the electrical circuit. The RF controller delivers thermal energy at a temperature of $65^{\circ} \mathrm{C}$ for 10 seconds, resulting in a maximum of 18 Watts of power delivered with each activation. A footswitch is used to deliver the activation at the appropriate time. The catheter contains a four-electrode basket on the distal tip and is marked at $5 \mathrm{~mm}$ increments. The basket is expanded using a handle to ensure adequate contact with the airway wall. Once an activation is delivered, the basket is collapsed and the catheter is withdrawn $5 \mathrm{~mm}$ to the next site of treatment. In this manner, the entire airway wall is treated from the distal to proximal direction without overlap. This process is repeated throughout all visual portions of the tracheobronchial tree in the lobe undergoing 
treatment. In general, patients will require 40-60 activations during each bronchoscopy. Airways previously treated should not be re-treated.

The most common complications during the procedure include bronchoconstriction, mucous hypersecretion, and minor bleeding related to superficial trauma. Patients should be monitored following the procedure, and treatment with bronchodilators in the immediate postprocedure setting is often needed. Spirometry and/or peak flow may be performed to ensure the patient is near the preprocedural baseline, though we do not routinely monitor this parameter at our institution postoperatively. In very severe asthmatics, particularly in the subgroup of older obese patients, noninvasive positive pressure ventilation may be helpful.

\section{Clinical safety and efficacy} Asthma Intervention Research (AIR) and Research in Severe Asthma (RISA) trials

The first clinical trial evaluating the safety of BT enrolled 16 patients with mild to moderate asthma. ${ }^{30}$ BT was well tolerated, with all procedure-related adverse events occurring in the week following the procedure. A majority of the adverse events were mild and transient and resolved spontaneously or with temporary increases in asthma medications. Although this trial was not powered to detect differences in outcomes, there was a significant reduction in airway hyperresponsiveness as measured by increased $\mathrm{PC}_{20}$ (provocative concentration causing a $20 \%$ decline in $\left.\mathrm{FEV}_{1}\right)(0.92 \mathrm{mg} / \mathrm{mL}$ at baseline, $4.75 \mathrm{mg} / \mathrm{mL}$ at 12 weeks, $5.45 \mathrm{mg} / \mathrm{mL}$ at 1 year, and $3.40 \mathrm{mg} / \mathrm{mL}$ at 2 years postprocedure). In addition, there was a significant improvement in symptom-free days ( $47 \%$ vs $73 \%, P=0.015)$ and peak expiratory flow rates, although these were measured only at 12 weeks following the procedure. There was no change in $\mathrm{FEV}_{1}$ during the 2 years of follow-up, though baseline spirometry in this cohort was normal with a mean $\mathrm{FEV}_{1}>80 \%$ of predicted. Chest $\mathrm{CT}$ was performed at 1 year and 2 years following BT with no evidence of bronchiectasis or parenchymal lung disease.

This was followed with the AIR trial, the first largescale, multicenter randomized controlled study of BT. ${ }^{31}$ A total of 112 patients with moderate to severe persistent asthma $\left(\mathrm{FEV}_{1} 60 \%-85 \%\right.$ of predicted and positive methacholine challenge test) were enrolled. There were no differences in prebronchodilator $\mathrm{FEV}_{1}$ percentage of predicted (72\%-74.3\% vs $75.8 \%-75.7 \%, P=0.28)$ or airway hyperresponsiveness between patients who underwent BT and the control group when compared to their prerandomization baseline. There was, however, a significant improvement in asthma symptoms as measured by symptom-free days $(40.6 \% \pm 39.7 \%$ vs $17.0 \% \pm 37.9 \%, P=0.005)$ and scores of the asthma control questionnaire (ACQ) (reduction, $1.2 \pm 1.0 \mathrm{vs}$ $0.5 \pm 1.0, P=0.001)$ and asthma quality of life questionnaire (AQLQ) ( $1.3 \pm 1.0$ vs $0.6 \pm 1.1, P=0.003)$. Additionally, there was a significant reduction in mild exacerbations (change in frequency per subject per week of $-0.16 \pm 0.37$ vs $0.04 \pm 0.29$, $P=0.005)$ and increases in morning peak expiratory flow rate (39.3 \pm 48.7 vs $8.5 \pm 44.2 \mathrm{~L} / \mathrm{min}, P=0.003)$ in patients treated with BT.

Shortly following the results of the AIR trial, the RISA trial was designed to evaluate the safety and efficacy of BT in patients with severe, symptomatic asthma. ${ }^{32}$ This smaller trial included 32 patients ( 15 randomized to BT) with severe persistent asthma as defined by uncontrolled symptoms despite high-dose ICS and LABA use. Prebronchodilator $\mathrm{FEV}_{1}$ was $62.9 \%$ of the predicted value in the BT group and $66.4 \%$ of predicted in the control group. In addition, 15 patients (eight in the BT group, seven in the control group) were using oral corticosteroids to maintain asthma control with a median dose of $15 \mathrm{mg} /$ day in BT group and $20 \mathrm{mg} /$ day among control patients. Following the procedure, all patients entered a phase where oral and inhaled corticosteroid dosing was held constant followed by a steroid weaning phase. There was a significant improvement in prebronchodilator $\mathrm{FEV}_{1}$ percentage predicted among BT subjects during the steroid stable phase $(14.9 \% \pm 17.4$ vs $-0.9 \% \pm 22.3, P=0.04)$, and a subsequent return closer to baseline following the steroid wean $(P=0.32)$. Furthermore, patients treated with BT had significant improvements in ACQ $(-1.04 \pm 1.03 \mathrm{vs}-0.13 \pm 1.00$ steroid stable phase, $P=0.02 ;-0.99 \pm 0.83$ vs $-0.22 \pm 0.78$ steroid wean phase, $P=0.01)$ and AQLQ $(1.21 \pm 1.05$ vs $0.15 \pm 0.75$ steroid stable phase, $P=0.003 ; 1.53 \pm 0.79$ vs $0.42 \pm 0.82$ steroid wean phase, $P=0.001)$ scores as well as reduced rescue beta agonist use (puffs/7 days, $-26.6 \pm 40.1$ vs $-1.5 \pm 11.7$ steroid stable phase, $P<0.05 ;-25.6 \pm 31.2$ vs $-6.1 \pm 12.4$ steroid wean phase, $P<0.05$ ).

\section{AIR-2 trial}

A common finding of both the AIR and RISA trials was a significant improvement in asthma symptoms and quality of life despite minimal, if any, improvements in pulmonary function. Given the unblinded nature of these trials, questions regarding the true efficacy of BT as compared to a potential placebo effect remained. To definitively answer these questions, the AIR-2 trial was performed using a multicenter, randomized, double-blind, sham-controlled design. ${ }^{33} \mathrm{~A}$ total of 288 subjects were enrolled in the study, with 190 patients 
undergoing BT. All patients had severe asthma based on the need for therapy with high-dose ICS and LABA, high ACQ scores, and low AQLQ scores. The primary outcome of interest was an increase in the AQLQ score from baseline.

Among patients treated with BT, the proportion of patients with a clinically meaningful increase in the AQLQ score of $\geq 0.5$ was significantly greater among those treated with BT when compared with patients who underwent sham bronchoscopy (79\% vs 64\%). Perhaps the most significant finding of the study was a significant reduction in the number of exacerbations (32\% risk reduction), emergency department visits ( $84 \%$ risk reduction), and days lost from school/work (66\% risk reduction) in patients treated with BT. Of note, patients treated with sham bronchoscopy did have an increase in the AQLQ score when compared to their prerandomization baseline. As a result, although the increase in AQLQ scores from baseline was significantly higher in the BT group compared to the control group ( $1.35 \pm 1.10$ vs $1.16 \pm 1.23)$, this placebo effect resulted in a small absolute difference between groups (0.19) and below a threshold that would be considered clinically meaningful. The results of these large clinical trials are summarized in Table 1.

\section{Long-term outcomes and safety}

The initial clinical trials of BT showed marked improvements in asthma-related quality of life and a reduction in the number of exacerbations but variable results on improvements in lung function. While promising, the longevity of these findings was not assessed in these studies. In addition, concerns regarding the long-term effects of thermal injury on lung function remained. Recently, the results from the long-term follow-up of patients enrolled in the AIR, RISA, and AIR-2 trials have sought to answer these questions.

From the original cohort of patients in the AIR trial, 45 patients treated with $\mathrm{BT}$ and 24 control patients consented to the extended follow-up study. ${ }^{34}$ BT patients were followed for an additional 4 years ( 5 years in total) while control patients were followed for an additional 2 years (3 years in total). Compared with the control group, patients who underwent BT had similar rates of respiratory adverse events, oral corticosteroid bursts, hospitalizations, and emergency department visits. Interestingly, patients treated with BT exhibited improvements in airway hyperresponsiveness out to 3 years (methacholine $\mathrm{PC}_{20}$ doublings in $\mathrm{BT}$ vs control: first year: $1.53 \pm 2.29$ vs $1.00 \pm 2.46, P=0.378$; second year: $1.21 \pm 2.99$ vs $-0.47 \pm 2.31, P=0.024$; third year: $1.31 \pm 2.96$ vs $-0.44 \pm 2.27, P=0025)$, arguing for the long-term efficacy of the procedure. Most importantly, there were no changes in measures of pulmonary function among patients treated with BT, and evaluation by serial chest X-rays revealed no clinically significant structural changes.

Follow-up evaluation of patients enrolled in the RISA trial was limited to subjects who underwent BT. ${ }^{35}$ Fourteen out of the fifteen patients who underwent BT from the original trial were followed for an additional 4 years (5 years total). Although the lack of data from the control group limits comparisons, there was a significant decrease in hospitalization and emergency department visits for respiratory symptoms in 1-5 years when compared to the year prior to BT. In addition, measures of pulmonary function showed no deterioration over the 5-year study period.

Patients enrolled in the AIR-2 study were also followed to evaluate the long-term effects of BT. ${ }^{36}$ Of the 192 subjects in the initial AIR-2 trial, 160 were followed for an additional 4 years. The most significant finding of the AIR-2 trial was a reduction in severe exacerbations and emergency department visits among those treated with BT. In the long-term follow-up study, the durability of these benefits was seen, with persistent reductions in these parameters throughout the follow-up period. Although patients in the control arm were not followed to make direct comparisons, a recent large-scale retrospective evaluation of patients with persistent asthma suggests that exacerbation rates remain constant despite continued high-intensity therapy with high doses of ICS and LABAs. ${ }^{37}$ In addition and in keeping with the findings of the previous long-term follow-up studies, no deterioration in lung function was noted. Furthermore, using high-resolution CT imaging, no significant structural changes were seen.

\section{BT in the management of severe asthma}

BT is currently approved for patients with severe persistent asthma who remain uncontrolled despite the use of an inhaled corticosteroid and LABA. The recently updated Global Initiative for Asthma (GINA) guidelines suggest that "for highlyselected adult patients with uncontrolled asthma despite use of recommended therapeutic regimens and referral to an asthma specialty center, bronchial thermoplasty is a potential treatment option" (Grade B evidence). ${ }^{38}$ BT is contraindicated in patients with a pacemaker, internal defibrillator, or any implantable electronic device. In addition, patients who cannot tolerate bronchoscopy or are unable to receive medications for sedation are not suitable candidates for BT.

Prior to consideration of BT, patients should undergo a thorough evaluation to ensure that the diagnosis of severe asthma is correct, treatment is optimized, and comorbid 
Table I Summary of the clinical trials and long-term follow-up of BT in asthma

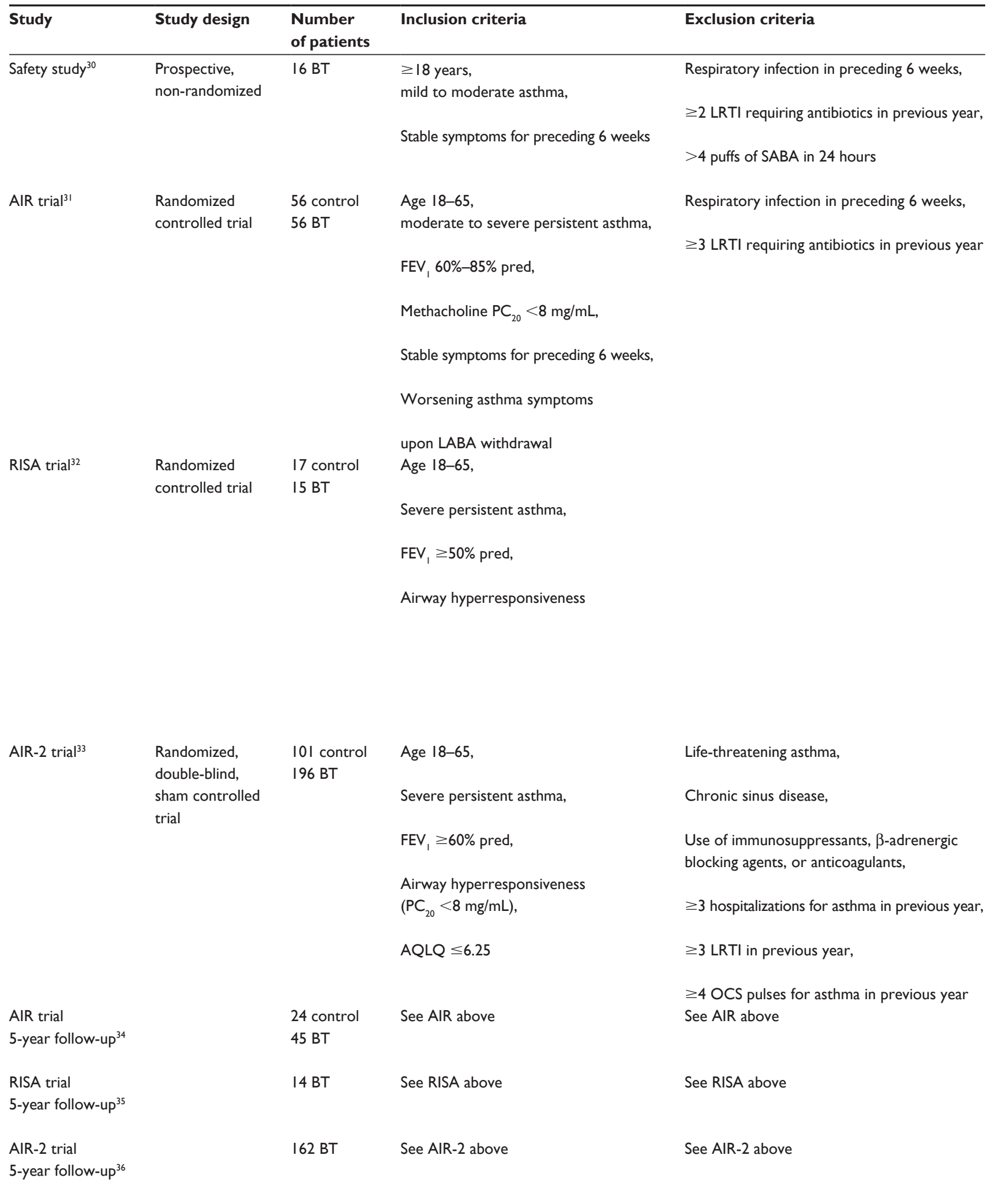

Abbreviations: BT, bronchial thermoplasty; LRTI, lower respiratory tract infection; SABA, short-acting beta agonist; PFT, pulmonary function testing; PEF, peak expiratory flow; AHR, airway hyperresponsiveness; $\mathrm{FEV}_{1}$, forced expiratory volume in I second; FVC, forced vital capacity; AIR, asthma intervention research; pred, predicted; $\mathrm{PC}_{20}$, provocative concentration causing a $20 \%$ decline in $\mathrm{FEV}$; $\mathrm{LABA}$, long-acting beta agonist; $\mathrm{ACQ}$, asthma control questionnaire; $\mathrm{AQLQ}$, asthma quality of life questionnaire; RISA, research in severe asthma; OCS, oral corticosteroid; ICS, inhaled corticosteroid; preBD, prebronchodilator; AIR-2, asthma intervention research-2; ED, emergency department. 


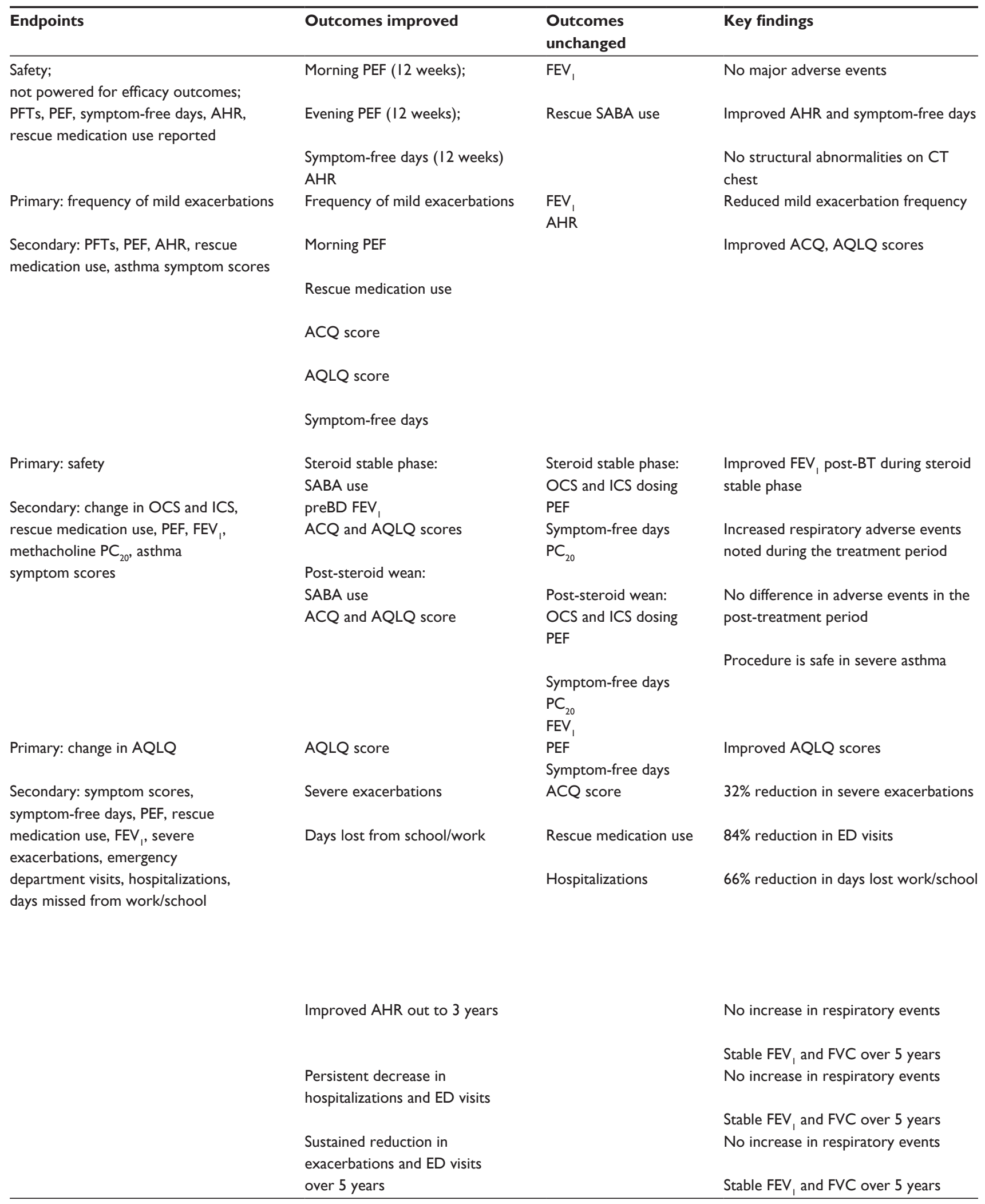


conditions are treated. In our practice, patients with severe asthma are evaluated in our Refractory Obstructive Lung Disease clinic. The first step in management involves a careful history and physical examination with attention toward signs or symptoms consistent with asthma. In addition, attention should be paid to symptoms that may suggest an alternative diagnosis, such as sarcoidosis, cystic fibrosis (CF), other non-CF bronchiectatic lung disease, alpha-1 antitrypsin deficiency, and chronic obstructive pulmonary disease (COPD).$^{39}$ As such, all patients receive full pulmonary function testing as well as a high-resolution CT scan of the lungs. In addition, laboratory evaluation including a complete blood count with differential, comprehensive metabolic panel, Ig levels, and alpha-1 antitrypsin level and genotype are obtained. If severe asthma is confirmed, the inhaler technique and adherence are evaluated rigorously, as nonadherence to medication is exceedingly common and interventions have been shown to improve asthma control. ${ }^{40}$ In addition, an evaluation for environmental triggers is performed with counseling on allergen avoidance and elimination.

An evaluation for comorbid conditions should be undertaken and treated as appropriate. Allergic rhinitis and sinusitis are frequently encountered in asthmatics and treatment with intranasal corticosteroids appears to improve asthma control. ${ }^{41,42}$ Treatment for gastroesophageal reflux disease (GERD) with oral proton pump inhibitors appears to have modest benefits in patients with symptomatic GERD but of little utility in "silent" reflux. ${ }^{43,44}$ Paradoxical vocal cord motion is often misdiagnosed as asthma, but it also frequently occurs concomitantly with asthma. ${ }^{45}$ If clinical suspicion is high, flexible laryngoscopy should be performed and, if confirmed, treatment by a specialized speech therapist is warranted.

In addition to high-dose ICS and LABA, we initiate therapy with a leukotriene-modifying agent if not already tried. For those patients with an allergic phenotype and elevated IgE levels, we initiate therapy with omalizumab. If therapy with omalizumab is unsuccessful or not indicated, we then consider BT. All patients undergo bronchoscopy for an airway examination as well as bronchoalveolar lavage and airway biopsy prior to consideration of BT. This allows for histologic evaluation of the ASM as well as ruling out any undiagnosed infectious disease. If increased ASM is confirmed, BT is offered as the next step in management (Figure 1).

Caution should be taken prior to proceeding with BT in patients with very severe airflow obstruction. The major clinical trials evaluating the safety and efficacy of BT excluded

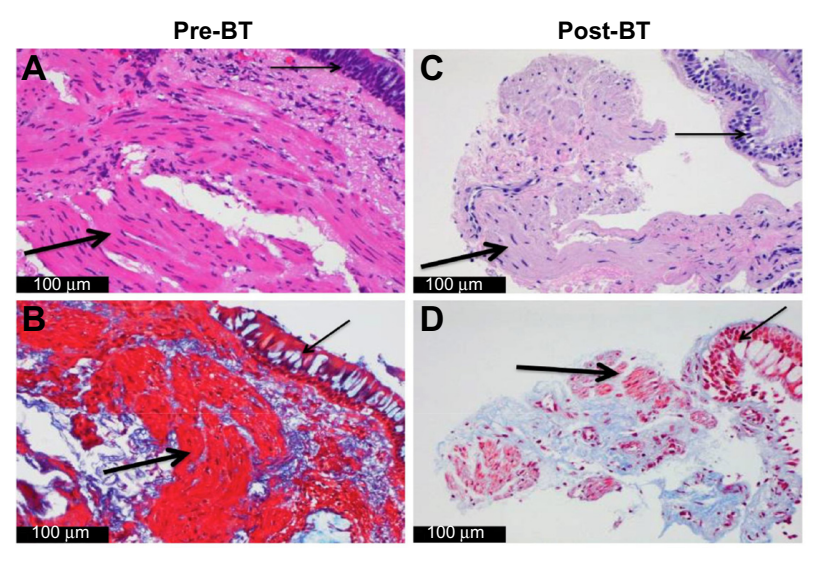

Figure I Airway histology before and after BT.

Notes: (A and $\mathbf{C})$ Hematoxylin and eosin and (B and $\mathbf{D})$ trichrome stain. Prominent ASM is seen in this patient with severe asthma, with significantly reduced ASM following treatment with BT. Thin arrow: airway epithelium; thick arrow: ASM. Abbreviations: BT, bronchial thermoplasty; ASM, airway smooth muscle.

the most severe asthmatics and general guidelines suggest that an $\mathrm{FEV}_{1}$ of less than $65 \%$ of predicted to be a relative contraindication. The largest trials, AIR and AIR-2, only excluded patients with a prebronchodilator $\mathrm{FEV}_{1}$ of less than $60 \%$ of predicted. However, the patients actually enrolled in the trials had a mean $\mathrm{FEV}_{1}$ of greater than $70 \%$ of predicted. The RISA trial evaluated patients with more severe asthma, but excluded patients with an $\mathrm{FEV}_{1}$ of less than $50 \%$ of predicted. We have previously reported our experience performing BT in patients with very severe asthma. ${ }^{46}$ The mean $\mathrm{FEV}_{1}$ in these eight patients was $51.8 \%$ of predicted, and included five patients with very severe asthma and a mean $\mathrm{FEV}_{1}$ of $37.4 \%$ of predicted. While patients required overnight inpatient observation frequently, there were no serious adverse events noted. Although we take caution when proceeding with BT in patients with very severe asthma and reduced $\mathrm{FEV}_{1}$, we do not feel it is a contraindication. In addition, we frequently employ overnight inpatient observation following the procedure for these patients.

\section{Future directions}

As a condition of the initial approval of BT, the United States FDA required two additional postapproval evaluations, including the 5-year follow-up results from the AIR-2 trial (available at http://www.accessdata.fda.gov/cdrh docs/pdf8/ P080032a.pdf). Another study, the Bronchial Thermoplasty in Severe Persistent Asthma (PAS2), is ongoing. ${ }^{47}$ With inclusion/exclusion criteria similar to that of the AIR-2 trial and a target enrollment of 300 patients, this trial will evaluate the frequency of severe exacerbations during 5 years of follow-up after BT treatment. In addition, registry data from patients undergoing BT is being collected. ${ }^{48}$ Together, these 
studies will seek to confirm the improvement in asthma control and reduced exacerbations with BT seen in the previous large clinical trials while also showing the long-term safety of the treatment.

The underlying mechanisms that lead to improvements in asthma control in patients treated with BT remain poorly understood. The intent of performing BT is to ablate the ASM, but this response is variable - as was seen in the feasibility study - and we have previously reported on a patient who exhibited persistent smooth muscle hyperplasia following treatment. ${ }^{49}$ Therefore, there are likely other mechanisms by which BT results in improved asthma symptoms, and much effort and interest is being taken to better understand the precise role of the ASM in the pathogenesis of asthma. ${ }^{50}$ Recently, a significant impairment in smooth muscle contraction following exposure to extreme temperature was noted. ${ }^{51}$ Recent evidence has also shown that the ASM plays an immunomodulatory role. ${ }^{52,53}$ In vivo, ASM cells probably produce chemokines and cytokines that play a role in leukocyte recruitment. ${ }^{54}$ In addition, they may produce antiinflammatory mediators. Furthermore, interactions between ASM cells and the extracellular matrix may play an important role in determining bronchomotor tone. ${ }^{55}$ These studies will not only help identify new therapeutic targets but also provide insights into the manner in which BT improves asthma control. More directly, several ongoing studies are evaluating the molecular, cellular, and histopathologic changes that occur in patients treated with BT, including one trial evaluating the potential effects of BT on the neuronal component of the bronchial mucosa. ${ }^{56,57}$

As has already been seen in trials of immunomodulatory agents in asthma, it is likely that certain phenotypes of asthma respond differently to BT. Therefore, it is critical to better understand the mechanisms that underlie the improvements seen with BT and use this information to identify subsets of patients who will have the largest clinical benefit. Ongoing trials are assessing the utility of imaging modalities including high-resolution CT scan and MRI to identify this subgroup. ${ }^{58,59}$ In addition, studies evaluating the use of blood and sputum biomarkers as well as airway histology to help identify these subsets of patients are underway. ${ }^{60,61}$

\section{Conclusion}

BT is a new therapy for patients with severe asthma. While the majority of therapies for asthma target the underlying inflammatory response, BT is the only therapy that specifically targets the ASM. Several clinical trials have demonstrated its safety and ability to improve quality of life and reduce exacerbations in patients with severe asthma. The exact mechanisms that underlie the improvements seen with BT remain poorly understood but are under intense study. Identifying the patients most likely to respond to this therapy is a critical next step and will be instrumental in determining the precise role of BT in the management of severe asthma.

\section{Disclosure}

Dr Laxmanan has no professional or financial interests to disclose. Dr Hogarth has served as a consultant to Boston Scientific and has lectured and received an unrestricted education grant.

\section{References}

1. American Lung Association, Epidemiology and Statistics Unit, Research and Program Services. Trends in Asthma Morbidity and Mortality; 2012. Available from: http://www.lung.org/finding-cures/ourresearch/trend-reports/asthma-trend-report.pdf. Accessed October 23, 2014.

2. World Health Organization (WHO). Asthma Fact Sheet No 307; 2013. Available from: http://www.who.int/mediacentre/factsheets/fs307/en/. Accessed October 23, 2014.

3. Godard P, Chanez P, Siraudin L, Nicoloyannis N, Duru G. Costs of asthma are correlated with severity: a 1-yr prospective study. Eur Respir J. 2002;19(1):61-67.

4. Ivanova JI, Bergman R, Birnbaum HG, Colice GL, Silverman RA, McLaurin K. Effect of asthma exacerbations on health care costs among asthmatic patients with moderate and severe persistent asthma. J Allergy Clin Immunol. 2012;129(5):1229-1235.

5. Moore WC, Bleecker ER, Curran-Everett D, et al. Characterization of the severe asthma phenotype by the National Heart, Lung, and Blood Institutes Severe Asthma Research Program. J Allergy Clin Immunol. 2007;119(2):405-413.

6. Chung KF, Wenzel SE, Brozek JL, et al. International ERS/ATS guidelines on definition, evaluation and treatment of severe asthma. Eur Respir J. 2014;43(2):343-373.

7. Moore WC, Meyers DA, Wenzel SE, et al; National Heart, Lung, and Blood Institute's Severe Asthma Research Program. Identification of asthma phenotypes using cluster analysis in the Severe Asthma Research Program. Am J Respir Crit Care Med. 2010;181(4): 315-323.

8. National Asthma Education and Prevention Program. Expert Panel Report 3 (EPR-3): Guidelines for the Diagnosis and Management of Asthma-Summary Report 2007. J Allergy Clin Immunol. 2007;120 (5 Suppl):S94-S138.

9. Dahlén SE, Malmström K, Nizankowska E, et al. Improvement of aspirin-intolerant asthma by montelukast, a leukotriene antagonist: a randomized, double-blind, placebo-controlled trial. Am J Respir Crit Care Med. 2002;165(1):9-14.

10. Robinson DS, Campbell D, Barnes PJ. Addition of leukotriene antagonists to therapy in chronic persistent asthma: a randomised double-blind placebo-controlled trial. Lancet. 2001;357(9273):2007-2011.

11. Virchow JC Jr, Prasse A, Naya I, Summerton L, Harris A. Zafirlukast improves asthma control in patients receiving high-dose inhaled corticosteroids. Am J Respir Crit Care Med. 2000;162(2 pt 1): 578-585.

12. Israel E, Cohn J, Dube L, Drazen JM. Effect of treatment with zileuton, a 5-lipoxygenase inhibitor, in patients with asthma. A randomized controlled trial. Zileuton Clinical Trial Group. JAMA. 1996;275(12): 931-936. 
13. Hanania NA, Alpan O, Hamilos DL, et al. Omalizumab in severe allergic asthma inadequately controlled with standard therapy: a randomized trial. Ann Intern Med. 2011;154(9):573-582.

14. Normansell R, Walker S, Milan SJ, Walters EH, Nair P. Omalizumab for asthma in adults and children. Cochrane Database Syst Rev. 2014;1: CD003559.

15. Busse WW, Israel E, Nelson HS, et al; Daclizumab Asthma Study Group. Daclizumab improves asthma control in patients with moderate to severe persistent asthma: a randomized, controlled trial. Am J Respir Crit Care Med. 2008;178(10):1002-1008.

16. Bel EH, Wenzel SE, Thompson PJ, et al; SIRIUS Investigators. Oral glucocorticoid-sparing effect of mepolizumab in eosinophilic asthma. N Engl J Med. 2014;371(13):1189-1197.

17. Ortega HG, Liu MC, Pavord ID, et al; MENSA Investigators. Mepolizumab treatment in patients with severe eosinophilic asthma. N Engl J Med. 2014;371(13):1198-1207.

18. Wenzel S, Ford L, Pearlman D, et al. Dupilumab in persistent asthma with elevated eosinophil levels. $N$ Engl J Med. 2013;368(26): $2455-2466$

19. Corren J, Lemanske RF, Hanania NA, et al. Lebrikizumab treatment in adults with asthma. $N$ Engl J Med. 2011;365(12):1088-1098.

20. Affonce DA, Lutchen KR. New perspectives on the mechanical basis for airway hyperreactivity and airway hypersensitivity in asthma. J Appl Physiol (1985). 2006;101(6):1710-1719.

21. James AL, Elliot JG, Jones RL, et al. Airway smooth muscle hypertrophy and hyperplasia in asthma. Am J Respir Crit Care Med. 2012; 185(10):1058-1064.

22. Bosse Y, Riesenfeld EP, Pare PD, Irvin CG. It's not all smooth muscle: non-smooth-muscle elements in control of resistance to airflow. Annu Rev Physiol. 2010;72:437-462.

23. An SS, Bai TR, Bates JH, et al. Airway smooth muscle dynamics: a common pathway of airway obstruction in asthma. Eur Respir J. 2007;29(5):834-860.

24. Panettieri RA Jr. Airway smooth muscle: immunomodulatory cells? Allergy Asthma Proc. 2004;25(6):381-386.

25. Danek CJ, Lombard CM, Dungworth DL, et al. Reduction in airway hyperresponsiveness to methacholine by the application of RF energy in dogs. J Appl Physiol (1985). 2004;97(5):1946-1953.

26. Brown RH, Wizeman W, Danek C, Mitzner W. In vivo evaluation of the effectiveness of bronchial thermoplasty with computed tomography. J Appl Physiol (1985). 2005;98(5):1603-1606.

27. Miller JD, Cox G, Vincic L, Lombard CM, Loomas BE, Danek CJ. A prospective feasibility study of bronchial thermoplasty in the human airway. Chest. 2005;127(6):1999-2006.

28. Mayse ML, Laviolette M, Rubin AS, et al. Clinical pearls for bronchial thermoplasty. J Bronchology Interv Pulmonol. 2007;14(2): 115-123.

29. Cox PG, Miller J, Mitzner W, Leff AR. Radiofrequency ablation of airway smooth muscle for sustained treatment of asthma: preliminary investigations. Eur Respir J. 2004;24(4):659-663.

30. Cox G, Miller JD, McWilliams A, Fitzgerald JM, Lam S. Bronchial thermoplasty for asthma. Am J Respir Crit Care Med. 2006;173(9): 965-969.

31. Cox G, Thomson NC, Rubin AS, et al; AIR Trial Study Group. Asthma control during the year after bronchial thermoplasty. $N$ Engl $J$ Med. 2007;356(13):1327-1337.

32. Pavord ID, Cox G, Thomson NC, et al; RISA Trial Study Group. Safety and efficacy of bronchial thermoplasty in symptomatic, severe asthma. Am J Respir Crit Care Med. 2007;176(12):1185-1191.

33. Castro M, Rubin AS, Laviolette M, et al; AIR2 Trial Study Group. Effectiveness and safety of bronchial thermoplasty in the treatment of severe asthma: a multicenter, randomized, double-blind, shamcontrolled clinical trial. Am J Respir Crit Care Med. 2010;181(2): $116-124$.

34. Thomson NC, Rubin AS, Niven RM, et al; AIR Trial Study Group. Long-term (5 year) safety of bronchial thermoplasty: asthma intervention research (AIR) trial. BMC Pulm Med. 2011;11:8.
35. Pavord ID, Thomson NC, Niven RM, et al; Research in Severe Asthma Trial Study Group. Safety of bronchial thermoplasty in patients with severe refractory asthma. Ann Allergy Asthma Immunol. 2013;111(5): 402-407.

36. Wechsler ME, Laviolette M, Rubin AS, et al; Asthma Intervention Research 2 Trial Study Group. Bronchial thermoplasty: long-term safety and effectiveness in patients with severe persistent asthma. J Allergy Clin Immunol. 2013;132(6):1295-1302.

37. Schatz M, Meckley LM, Kim M, Stockwell BT, Castro M. Asthma exacerbation rates in adults are unchanged over a 5 -year period despite high-intensity therapy. J Allergy Clin Immunol Pract. 2014;2(5): 570-574. e1.

38. Global Initiative for Asthma (GINA) [homepage on the Internet]. Global Strategy for Asthma Management and Prevention. Available from: http://www.ginasthma.org/local/uploads/files/GINA_Report_2015.pdf. Accessed March 18, 2015.

39. Robinson DS, Campbell DA, Durham SR, et al; Asthma and Allergy Research Group of the National Heart and Lung Institute. Systematic assessment of difficult-to-treat asthma. Eur Respir J. 2003;22(3): 478-483.

40. Gamble J, Stevenson M, Heaney LG. A study of a multi-level intervention to improve non-adherence in difficult to control asthma. Respir Med. 2011;105(9):1308-1315.

41. Adams RJ, Fuhlbrigge AL, Finkelstein JA, Weiss ST. Intranasal steroids and the risk of emergency department visits for asthma. J Allergy Clin Immunol. 2002;109(4):636-642.

42. Corren J, Manning BE, Thompson SF, Hennessy S, Strom BL. Rhinitis therapy and the prevention of hospital care for asthma: a case-control study. J Allergy Clin Immunol. 2004;113(3): 415-419.

43. American Lung Association Asthma Clinical Research Centers; Mastronarde JG, Anthonisen NR, et al. Efficacy of esomeprazole for treatment of poorly controlled asthma. $N$ Engl J Med. 2009;360(15): $1487-1499$.

44. Littner MR, Leung FW, Ballard ED 2nd, Huang B, Samra NK; Lansoprazole Asthma Study Group. Effects of 24 weeks of lansoprazole therapy on asthma symptoms, exacerbations, quality of life, and pulmonary function in adult asthmatic patients with acid reflux symptoms. Chest. 2005;128(3):1128-1135.

45. Yelken K, Yilmaz A, Guven M, Eyibilen A, Aladag I. Paradoxical vocal fold motion dysfunction in asthma patients. Respirology. 2009;14(5): 729-733.

46. Doeing DC, Mahajan AK, White SR, Naureckas ET, Krishnan JA, Hogarth DK. Safety and feasibility of bronchial thermoplasty in asthma patients with very severe fixed airflow obstruction: a case series. J Asthma. 2013;50(2):215-218.

47. Boston Scientific Corportation. Bronchial Thermoplasty in Severe Persistent Asthma (PAS2); 2014. Available from: https://clinicaltrials. gov/show/NCT01350336. Accessed December 3, 2014. [NML Identifier: NCT 01350336].

48. Boston Scientific Corporation. Registry of Bronchial Thermoplasty (BT) Procedures EMEA BT Registry; 2014. Available from: https:// clinicaltrials.gov/ct2/show/NCT02104856. Accessed December 4, 2014. [NLM Identifier: NCT02104856].

49. Doeing DC, Husain AN, Naureckas ET, White SR, Hogarth DK. Bronchial thermoplasty failure in severe persistent asthma: a case report. J Asthma. 2013;50(7):799-801.

50. Dowell ML, Lavoie TL, Solway J, Krishnan R. Airway smooth muscle: a potential target for asthma therapy. Curr Opin Pulm Med. 2014;20(1):66-72.

51. Dyrda P, Tazzeo T, DoHarris L, et al. Acute response of airway muscle to extreme temperature includes disruption of actin-myosin interaction. Am J Respir Cell Mol Biol. 2011;44(2):213-221.

52. Panettieri RA Jr, Kotlikoff MI, Gerthoffer WT, et al; National Heart, Lung, and Blood Institute. Airway smooth muscle in bronchial tone, inflammation, and remodeling: basic knowledge to clinical relevance. Am J Respir Crit Care Med. 2008;177(3):248-252. 
53. Solway J, Irvin CG. Airway smooth muscle as a target for asthma therapy. N Engl J Med. 2007;356(13):1367-1369.

54. Lazaar AL, Albelda SM, Pilewski JM, Brennan B, Pure E, Panettieri RA Jr. $\mathrm{T}$ lymphocytes adhere to airway smooth muscle cells via integrins and CD44 and induce smooth muscle cell DNA synthesis. $J$ Exp Med. 1994;180(3):807-816.

55. Freyer AM, Billington CK, Penn RB, Hall IP. Extracellular matrix modulates beta2-adrenergic receptor signaling in human airway smooth muscle cells. Am J Respir Cell Mol Biol. 2004;31(4): 440-445.

56. Arcispedale Santa Maria Nuova-IRCCS. Bronchial Thermoplasty: Effect on Neuronal and Chemosensitive Component of the Bronchial Mucosa (BT-ASMN); 2014. Available from: https://clinicaltrials.gov/ct2/ show/NCT01839591. Accessed December 6, 2014. [NLM Identifier: NCT01839591].

57. Academisch Medisch Centrum-Universiteit van Amsterdam (AMC-UvA). Unravelling Targets of Therapy in Bronchial Thermoplasty in Severe Asthma (TASMA); 2014. Available from: https://clinicaltrials. gov/ct2/show/NCT02225392. Accessed December 8, 2014. [NLM Identifier: NCT02225392].
58. Xemed LLC. Bronchial Thermoplasty for Severe Asthmatics Guided by HXe MRI (HXe-BT); 2014. Available from: https://clinicaltrials.gov/ct2/ show/NCT01832363. Accessed December 7, 2014. [NLM Identifier: NCT0182363].

59. Washington University School of Medicine. A Prospective Observational Study of Biopredictors of Bronchial Thermoplasty Response in Patients with Severe Refractory Asthma (BTR Study); 2014. Available from: https://clinicaltrials.gov/ct2/show/NCT01185275. Accessed December 7, 2014. [NLM Identifier: NCT01185275].

60. Fundació Institut de Recerca de l'Hospital de la Santa Creu i Sant Pau. Study of Physiopathological Mechanisms and Results of Treatment with Bronchial Thermoplasty in Severe Asthma; 2014. Available from: https://clinicaltrials.gov/ct2/show/NCT01974921. Accessed December 5, 2014. [NLM Identifier: NCT01974921].

61. University of Leicester (UK). Bronchial Thermoplasty Study: An Observational Study Examining Airway Remodelling and Repair in Patients with Severe Persistent Asthma Treated with Bronchial Thermoplasty; 2014. Available from: http://www.isrctn.com/ISRCTN94263922. Accessed December 8, 2014. [NLM Identifier: ISRCTN94263922].

\section{Publish your work in this journal}

The Journal of Asthma and Allergy is an international, peer-reviewed open-access journal publishing original research, reports, editorials and commentaries on the following topics: Asthma; Pulmonary physiology; Asthma related clinical health; Clinical immunology and the immunological basis of disease; Pharmacological interventions and

\section{Dovepress}

new therapies. Issues of patient safety and quality of care will also be considered. The manuscript management system is completely online and includes a very quick and fair peer-review system, which is all easy to use. Visit http://www.dovepress.com/testimonials.php to read real quotes from published authors. 